\title{
不同干旱土壤条件下杨树的耗水规律 及水分利用效率研究
}

\author{
杨建伟 ${ }^{12}$ 梁宗锁 ${ }^{1} 3^{*} \quad$ 韩莈莲 $^{1} \quad$ 孙 $\quad$ 群 $^{3} \quad$ 崔浪军 $^{13}$ \\ （1 中国科学院水利部水土保持研究所, 陕西杨凌 712100) （2 南阳师范学院, 河南南阳 473061) \\ (3 西北农林科技大学生命科学学院, 陕西杨凌 712100)
}

\begin{abstract}
摘 要 在适宜土壤水分 $\left(70 \% \theta_{f}\right)$ 、中度干旱 $\left(55 \% \theta_{f}\right)$ 和严重干旱 $\left(40 \% \theta_{f}\right) 3$ 种土壤水分条件下研究杨树 (Populus simonii) 的耗水特性和水分利用特征。结果表明，随着土壤含水量的下降，杨树叶水势、相对含水量 $(R W C)$ 、生长速 率、光合速率及单叶水分利用效率 (WUE) 显著下降; 在适宜水分和中度干旱条件下 杨树的快速生长和干物质迅速 积累时期主要集中在 5 6 月, 严重干旱下快速生长时期和干物质积累主要集中在 5 月; 杨树总耗水量和总生物量 的大小顺序为 适宜水分 > 中度干旱 > 严重干旱; WUE 则表现出中度干旱下最高, 严重干旱下最低 杨树在适宜水 分下的日、旬、月耗水量明显高于中度干旱和严重干旱处理;杨树在适宜水分、中度干旱和严重干旱条件下的最高 耗水月分别在 6 7 月 最高旬耗水量分别在 7 月中旬、上旬和 6 下旬; 在中度水分亏缺和严重水分亏缺下的最高耗 水日出现的时间比适宜水分下的最高耗水日提前 $1 \sim 2$ 个月以上。一天中的最大耗水高峰随着杨树生育期和土壤 含水量的不同而有明显差异。研究结果表明 杨树不具备耐旱植物的特征, 因此在黄土高原缺水地区不适宜大面 积栽植，只能用于水分条件较好的立地条件下造林。
\end{abstract}

关键词 杨树 土壤水分 耗水量 水分利用率

\section{WATER USE EFFICIENCY AND WATER CONSUMPTION CHARACTERISTICS OF POPLAR UNDER SOIL DROUGHT CONDITIONS}

\author{
YANG Jian-Wei ${ }^{1}{ }^{2}$ LIANG Zong-Suo ${ }^{1,3}$ HAN Rui-Lian ${ }^{1}$ SUN Qun ${ }^{3}$ and CUI Lang-Jun ${ }^{1,3}$ \\ (1 China Institute of Soil and Water Conservation, Chinese Academy of Sciences and Ministry of Water Resources , Yangling , Shaanxi 712100 , China) \\ (2 Nan Yang Teacher's College, Nanyang, Henan 473061 , China) \\ (3 College of Life Science, Northwest Sci-Tech University of Agriculture and Forestry, Yangling, Shaanxi 712100 , China)
}

\begin{abstract}
We studied water consumption and water use characteristics of poplar under three different soil water regimes : adequate soil water, medium drought, and severe drought conditions, which were about $70 \%$, $55 \%$ and $40 \%$ of field capacity. The results showed that water potential , water content, photosynthesis rate, water use efficiency of leaves, and plant growth rates decreased significantly with decreases in soil water content. At levels of adequate soil water content and medium drought, fast growth and biomass accumulation of poplar occurred during May and June, whereas this occurred during May only under severe drought conditions. Total water consumption and biomass growth were greatest under adequate soil water conditions, decreased under medium drought, and was lowest under severe drought conditions. Total water use efficiency was highest under medium drought conditions and lowest under severe drought. Patterns of water consumption on a daily rate, every 10 days, and monthly rates were different under the three soil water treatments. For all three treatments , the highest monthly water consumption occurred in July and then June. The highest water consumption over a ten-day period was in the second ten days of July, the first ten days of July, and the last ten days of June for the three treatments, respectively. The highest water consumption day of the medium and severe drought treatments occurred one or two months earlier than the adequate soil water treatment. The time of day when the greatest water consumption occurred changed throughout the life span of the poplar and under the differnt soil water treatments. From our results, we conclude that poplar does not have characteristics of drought resistance plants ; hence, we do not recommend that this species is planted over a wider range in the loess plateau.
\end{abstract}

Key words Populus simonii, Water consumption, Soil water content, Water use efficiency 
在我国北方，特别是西北的干旱、半干旱地区， 植物只能依靠当年的降水和灌溉维持生长, 在没有 灌溉条件的地方，杨树 (Populus simonii)生长受到了 严重的抑制，从而限制了这种优良树种的经济和生 态效益的发挥(侯庆春等, 2000)。长期以来, 关于杨 树的研究有许多报道(刘雅荣等, 1983;刘奉觉等, 1991 ;湖新生等, 1997; 卜基保, 1997;曾凡江等,2000； 巨关升等,2000 ;褚作旺等,2003; 贾黎明等 ,2004; 李 洪建等,2000; 高健等,2002), 但主要集中在华北和 东北地区, 而对于严重缺水的黄土高原常见树种杨 树研究较少, 尤其是缺乏对杨树在不同土壤干旱下 的生长、水分关系和水分利用特征的系统研究，从而 限制了杨树在我国不同区域和不同立地条件下的合 理配置, 导致目前在黄土高原形成了大面积低产、林 分结构脆弱的 小老树”。本文针对当前黄土高原大 面积人工造林树种选择所面临的问题, 以黄土高原 已经大面积栽植的杨树为试验材料, 利用陕北黄土 高原的黄绵土，在人工控制土壤水分条件下 模拟该 树种生长环境的土壤水分条件, 系统研究在不同土 壤水分条件下该树种的耗水规律及水分利用效率， 以期为黄土高原地区选择造林树种提供科学的理论 依据。

\section{1 材料和方法}

\section{1 试验材料与水分处理}

本试验采用黄土高原常见的造林树种 $84 \mathrm{~K}$ 杨 树' 为试验材料, 由中国科学院安塞生态试验站提供 一年生的实生苗。试验用土为安塞生态站的黄绵 土, 田间持水量为 $21.5 \%$ 。设置 3 种土壤含水量水 平 适宜水分、中度水分亏缺、严重水分亏缺，即分 别是田间持水量 $\left(\theta_{f}\right)$ 的 $70 \% 、 55 \% 、 40 \%$ (分别为重 量含水率), 各处理分别设置 $5 \sim 6$ 盆重复, 每盆栽植 $3 \sim 4$ 株苗, 待成活后选择大小基本一致的苗木保留 2 株，于 2001 年 3 月 6 日植入口径 $20 \mathrm{~cm}$ 、高 $30 \mathrm{~cm}$ 的生长钵内, 放置在中国科学院水土保持研究所的 可移动模拟干旱防雨棚内培养, 雨天用防雨棚遮雨, 晴天露地生长。从移栽次日开始, 每天定时用重量 法控制土壤含水量, 并加水补充其蒸腾损失, 为排除 土壤蒸发用塑料薄膜覆盖盆面裸土。每次用量杯记 录加水量, 整个试验持续至 10 月中旬结束, 历时 210 d。

\section{2 测定项目及方法}

1.2.1 水分状况与水分利用率 (WUE) 的测定 叶片水势 $\left(\psi_{w}\right)$ 用小液流法测定: 每月选择晴朗
天气, 于上午 9:00 采样, 并迅速带回实验室, 每样重 复 3 次测定 旪片含水量 $(R W C)$ 选取功能叶用烘干 称重法测定, 并将叶片干重计入总生物量; 单叶 $W U E=$ 光合速率 $\left(P_{n}\right)$ /蒸腾速率 $\left(T_{r}\right)$ 谂 $W U E=$ 总 生物量/生长季耗水总量。

\subsection{2 $P_{n}$ 和 $T_{r}$ 的测定}

在生长季每月选择晴朗无云的天气,于早上 9:00用 LI-6400 型便携式光合仪测定, 每处理选 5 6 片功能叶 取其平均值。

\section{2 .3 新生枝条生长速率}

每隔 $15 \mathrm{~d}$ 定时用毫米刻度尺定位测量新生枝 条长度的变化, 计算单位时间的生长速率, 干物质月 增量的测定，从移栽后每月选取有代表性枝条烘干 称重, 并同时将枝条干重计入总生物量, 然后对整株 新生枝条进行估算，总生物量为试验结束时苗木的 总干重减去栽前干重, 栽前干重的测定是选取与将 要移栽的苗木大小一致的苗木 10 株, 烘干称重, 取 其平均值。

\subsection{4 耗水量的测定}

每天定时称盆重,在排除土壤蒸发和苗重的增 量后, 以盆重的减少量为树种的当日耗水量, 并加水 补充至设定土壤含水量;旬耗水量为当月每旬耗水 量总和;月耗水量为当月每日耗水量的总和计算 搃 耗水量为整个生长季每天加水量与移栽苗木之前和 起苗后土壤含水量变化之和; 耗水日进程采用称重 法测定，从早上 7:00 到下午 19:00,每隔 $2 \mathrm{~h}$ 称盆重 1 次, 以盆重的减少量为树种在该时间段内的耗水 量, 并同时加水补充至设定土壤含水量。

\section{2 结果与分析}

\section{1 土壤干旱对杨树叶 $\psi_{w}$ 及叶片组织含水量的影 响}

叶片 $\psi_{w}$ 是反映植物缺水最敏感的指标之一， 叶含水量则可以直接反映植物体内的水分状况，从 图 1 2 可以看出, 试验期内杨树在适宜水分、中度 干旱和严重干旱条件下, 其叶片 $\psi_{w}$ 从 $-0.8 \mathrm{MPa}$ 分 别下降至 $-1.2 、-1.6$ 和 $-2.6 \mathrm{MPa}$, 在严重干旱条 件下下降幅度最大, 适宜水分下水势下降幅度最小。

杨树的叶片组织含水量，在适宜水分下从 $60 \%$ 降至 59\% ,下降 $1.7 \%$, 在中度亏缺下从 $59.4 \%$ 降至 $56 \%$,下降 $5.7 \%$, 在严重亏缺下则从 $59.2 \%$ 降至 $52 \%$ ，下降 $12.2 \%$ ，下降幅度最大。以上结果说明， 在试验期间, 从春季到秋季, 随着季节的变化, 杨树 叶片含水量是逐渐降低的, 而且土壤含水量越低, 下 
降幅度越大。

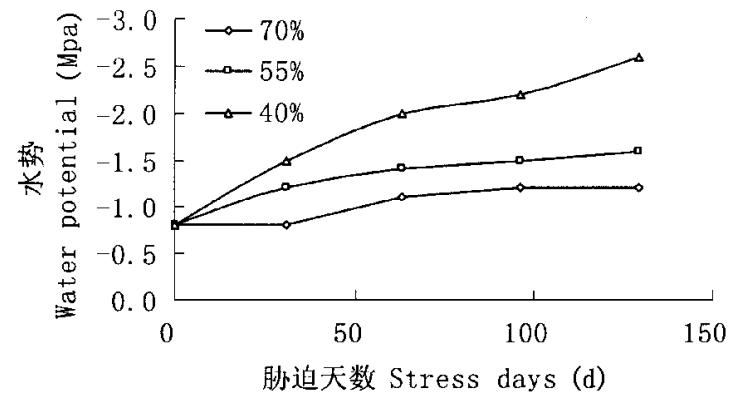

图 1 不同土壤水分条件下杨树叶片 $\psi_{w}$ 变化

Fig. 1 Changes of water potential of Populus simonii leaf under different soil water stress

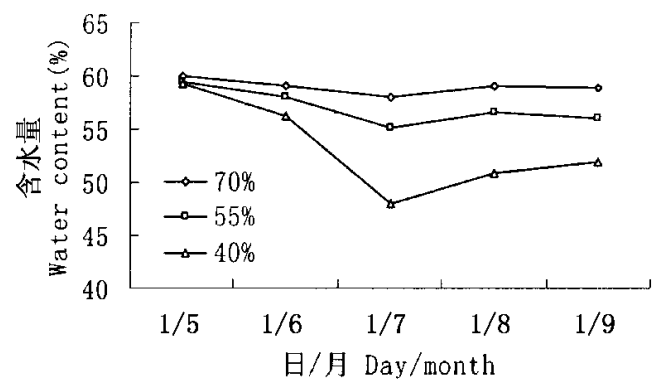

图 2 不同土壤水分条件下杨树叶片含水量变化

Fig.2 Changes of water content of Populus simonii leaf under different soil water stress

\section{2 土壤干旱对杨树枝条生长速率和干物质积累} 的影响

在不同土壤干旱下, 杨树的生长速率 (图 3 ) 及 干重净增量 (图 4) 明显不同, 其生长速率和干重净 增量均表现为适宜水分 > 中度干旱 > 严重干旱。在 适宜水分和中度干旱条件下，其枝条生长速率呈 S" 形生长曲线 , 3 4 月生长较慢 $A$ 月下旬以后生长急 剧加快, 7 月以后生长极为缓慢; 杨树在整个生长季 中枝条快速生长期和干物质增加主要集中在 $5 \sim 6$ 月, 除适宜水分下的干重在 8 月略有上升外, 其余干 重增加量 7 月以后逐渐下降, 9月降至最低, 严重干 旱下的干物质增加主要集中在 5 月。与适宜水分处 理相比, 在中度干旱下新枝伸长量下降 $5.2 \%$,干重 下降 $27.84 \%$, 在严重干旱下杨树新枝伸长量下降 $71.92 \%$,干重下降 $72.94 \%$ 。很显然在严重干旱条 件下杨树枝条生长速率和干重净增量下降显著, 杨 树新枝伸长的最快时期在 5 6 月,7 月以后新枝伸 长量几乎不再增加, 而在 3 6 月正是黄土高原雨季 来临前土壤水分最为亏缺的时期，这可能是黄土高 原人工杨树林生长发育不良，而形成大面积 小老 树”的主要原因。

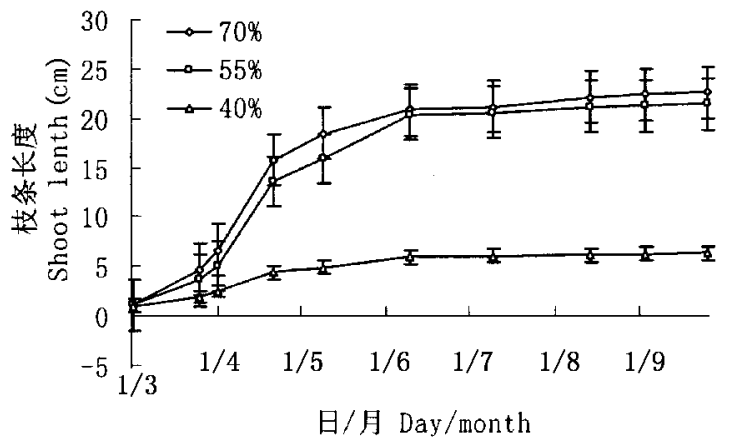

图 $33 \sim 10$ 月杨树枝条生长速率

Fig.3 Growth rate of Populus simonii in growth season

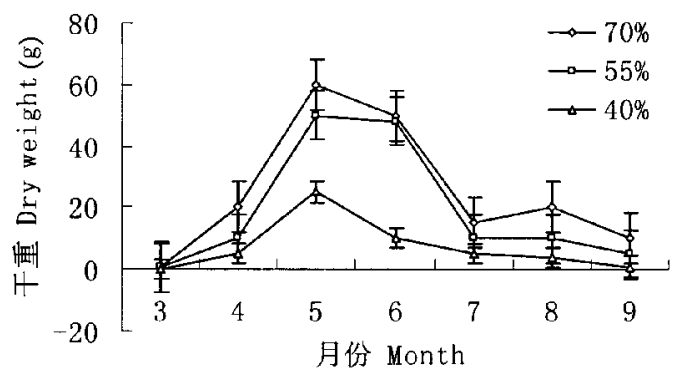

图 4 3 9 月杨树枝条干重增量

Fig.4 Biomass increment rate of Populus simonii branches in growth season

2.3 不同土壤水分状况对杨树光合速率、蒸腾速率 及单叶 $W U E$ 的影响

在生长季各月的晴朗天气 利用 LI-6400 型便携 式光合仪测定杨树的光合速率和蒸腾速率, 测定结 果发现 杨树的光合速率在生长初期 $5 \sim 6$ 月最高 ,7 月以后逐渐下降,末期降至最低; 在整个生长季中, 不同月以及在同一月的 3 种处理之间所测定的蒸腾 速率变化规律不明显; 单叶水平上的 $W U E$ 在生长 中期时 (7 8 月)略高于其它时期。此外, 在同一测 定时间内, 随着土壤含水量的降低 杨树的光合速率 及单叶 $W U E$ 也随着下降, 其顺序在各月均表现为 适宜水分 > 中度干旱 > 严重干旱, 即在适宜水分下 光合速率及 $W U E$ 均为最高, 严重干旱下最低。以 生长初期 (5 月 10 日)、中期 (7 月 31 日)和末期(9 月 15 日)所测结果为例列表如下 (表 1)。

2.4 土壤水分含量对杨树的日、旬、月耗水量及各 月耗水日进程的影响

2.4 .1 土壤水分含量对杨树的日、旬、月耗水量影 响

在 3 10 月整个生长季中杨树的日耗水变化为 多峰波动状曲线 (图 5), 其中曲线的峰值和低谷分 别为晴天和阴雨天的耗水量，在适宜土壤水分下杨 树的日平均耗水量是中度水分亏缺和严重水分亏缺 
表 15 月 10 日、7 月 31 日和 9 月 15 日杨树在 3 种土壤水分下光合速率 $\left(P_{n}\right)$ 、蒸腾速率 $\left(T_{r}\right)$ 及单叶 WUE 的变化

Table 1 The change of photosynthesis rate, transpiration rate and water use efficiency of Populus simonii leaf on May 10 , July 31 and September 15

\begin{tabular}{|c|c|c|c|c|c|c|c|c|c|}
\hline \multirow{2}{*}{$\begin{array}{c}\text { 处理 } \\
\text { Treatment }\end{array}$} & \multicolumn{3}{|c|}{$\begin{array}{l}\text { 生长初期 Early season of growth } \\
\text { (5月 } 10 \text { 日 May 10) }\end{array}$} & \multicolumn{3}{|c|}{$\begin{array}{c}\text { 生长中期 Medium season of growth } \\
\text { (7 月 } 31 \text { 日 July 31) }\end{array}$} & \multicolumn{3}{|c|}{$\begin{array}{l}\text { 生长末期 Late season of growth } \\
\text { (9月 } 15 \text { 日 September 15) }\end{array}$} \\
\hline & $\begin{array}{l}P_{n}(\mu \mathrm{mol} \\
\left.\mathrm{m}^{-2} \mathrm{~s}^{-1}\right)\end{array}$ & $\begin{array}{l}T_{r}(\mathrm{mmol} \\
\left.\mathrm{m}^{-2} \mathrm{~s}^{-1}\right)\end{array}$ & $\begin{array}{l}\text { WUE } \\
\left(P_{n} / \mathrm{E}\right)\end{array}$ & $\begin{array}{l}P_{n}(\mu \mathrm{mol} \\
\left.\mathrm{m}^{-2} \mathrm{~s}^{-1}\right)\end{array}$ & $\begin{array}{l}T_{r}(\mathrm{mmol} \\
\left.\mathrm{m}^{-2} \mathrm{~s}^{-1}\right)\end{array}$ & $\begin{array}{c}\text { WUE } \\
\left(P_{n} / T_{r}\right)\end{array}$ & $\begin{array}{l}P_{n}(\mu \mathrm{mol} \\
\left.\mathrm{m}^{-2} \mathrm{~s}^{-1}\right)\end{array}$ & $\begin{array}{l}T_{r}(\mathrm{mmol} \\
\left.\mathrm{m}^{-2} \mathrm{~s}^{-1}\right)\end{array}$ & $\begin{array}{c}\text { WUE } \\
\left(P_{n} / T_{r}\right)\end{array}$ \\
\hline $70 \% \theta_{f}$ & $11.8^{\mathrm{aA}}$ & $2.6^{\mathrm{a}}$ & $4.29^{\mathrm{a}}$ & $10.75^{\mathrm{aA}}$ & $1.89^{\mathrm{a}}$ & $5.68^{\mathrm{a}}$ & $5.13^{\mathrm{aA}}$ & $1.85^{\mathrm{a}}$ & $2.7^{\mathrm{a}}$ \\
\hline $55 \% \theta_{f}$ & $10.73^{\mathrm{abAB}}$ & $3.11^{\mathrm{a}}$ & $3.45^{\mathrm{a}}$ & $10.2^{\mathrm{aA}}$ & $1.94^{\mathrm{a}}$ & $5.26^{\mathrm{a}}$ & $3.87^{\mathrm{bB}}$ & $1.48^{\mathrm{a}}$ & $2.61^{\mathrm{a}}$ \\
\hline $40 \% \theta_{f}$ & $9.57^{\mathrm{bB}}$ & $2.93^{\mathrm{a}}$ & $3.26^{\mathrm{a}}$ & $8.47^{\mathrm{bA}}$ & $2.31^{\mathrm{a}}$ & $3.67^{\mathrm{a}}$ & $2.55^{\mathrm{cC}}$ & $1.35^{\mathrm{a}}$ & $1.89^{\mathrm{b}}$ \\
\hline
\end{tabular}

相同字母不显著 小写为 $\alpha=0.05$ 水平 大写为 $\alpha=0.01$ 水平 ( SSR 检验) The same letter indicates insignificant Small letter means $\alpha=0.05$ and capital letter means $\alpha=0.01$ (SSR test)

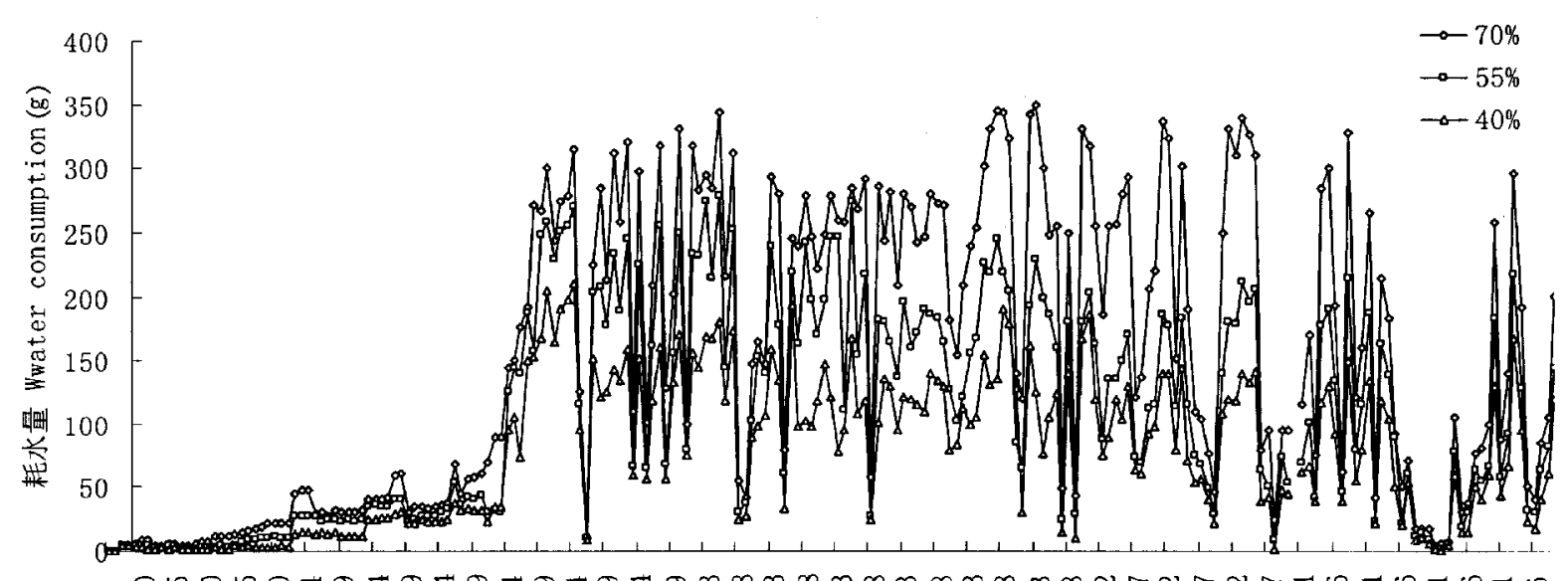

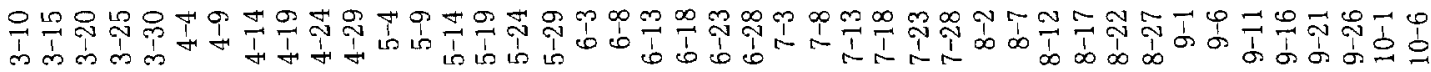

月-日 Month-day

图 53 种土壤水分下杨树 3 10 月日耗水变化

Fig.5 Changes of diurnal water consumption of Populus simonii under three soil water stress

耗水量的 1.6 和 2.7 倍。而在 3 种土壤水分下,杨 树的最高耗水日分别在 7 月 23 日、 6 月 5 日和 5 月 14 日, 在中度干旱和严重干旱下的最高耗水日出现 的时间比适宜水分下的最高耗水日提前 $1 \sim 2$ 个月 以上。

表 2 的结果显示，杨树的耗水量从 5 月上旬逐 渐上升。在 $70 \% 、 55 \%$ 和 $40 \% 3$ 种土壤水分下的最 高旬耗水量分别在 7 月中旬、 7 月上旬和 6 月下旬， 其中在 9 月中、下旬的耗水量比 10 月低是与当时的 连阴雨天气有关。杨树在 3 种土壤水分条件下的月 耗水高峰在 $5 \sim 8$ 月, 在 3 种土壤水分条件下的最高 月耗水量分别在 6 7 月 (图 6)。可以看出土壤含 水量越低 杨树的最高旬耗水量和最高月耗水量出 现的越早。而在 3 种不同的土壤水分下比较, 杨树 的日耗水量、旬耗水量、月耗水量大小顺序均为适宜 水分 > 中度干旱 > 严重干旱, 方差分析显示其差异 性达到极显著水平。在整个生长季中 $3 \sim 4$ 月、 $9 \sim$ 10 月耗水处于低水平, 这与早春枝条尚未展开或秋 季气温低、生理功能衰退有关。

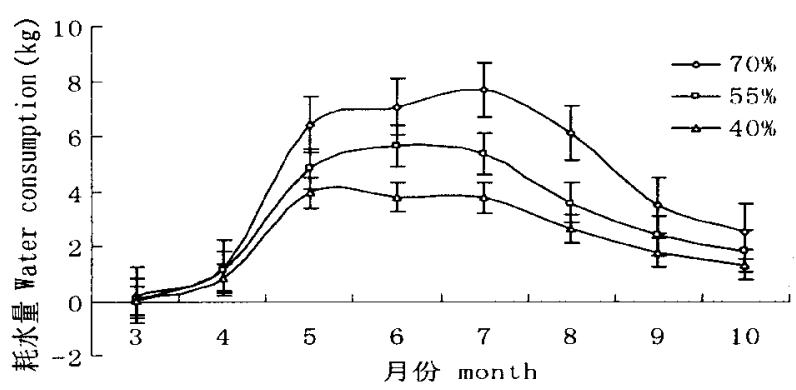

图 63 种土壤水分下杨树生长季逐月耗水变化 Fig.6 Water consumption of Populus simonii in a month under three soil water stress

\subsection{2 土壤水分含量对杨树各月耗水日进程的影响}

从 5 月树叶完全展开到 10 月上旬落叶前为止， 杨树在不同的测定日 (图 7) , 其耗水进程有明显差 异。5月 (图 7a) 的耗水日变化 3 种处理全部为单 峰曲线, 耗水量高峰在 11:00 15:00 之间; 6月 3 种 处理的耗水量 (图 7b) 与 5 月相比日耗水高峰提前 $2 \sim 3 \mathrm{~h}$, 其耗水量高峰在 9:00 12:00 之间; 在 7 月 (图 7c) 杨树的 3 种处理日耗水呈双峰曲线, 其耗 水的两个高峰分别在 $12: 00$ 左右和 $16: 00$ 左右; 杨 
表 $23 \sim 10$ 月杨树每旬耗水量 $\left(\mathrm{kg}\right.$ 每株 $\left.^{-1}\right)$

Table 2 Water consumption in a period of ten days of Populus simonii under different soil water stress $\left(\mathrm{kg}\right.$. plant ${ }^{-1}$ )

\begin{tabular}{|c|c|c|c|c|c|c|c|c|c|}
\hline \multirow{3}{*}{$\begin{array}{l}\text { 月份 } \\
\text { Month }\end{array}$} & \multicolumn{9}{|c|}{ 土壤水分 Soil water } \\
\hline & \multicolumn{3}{|c|}{$70 \% \theta_{f}$} & \multicolumn{3}{|c|}{$50 \% \quad \theta_{f}$} & \multicolumn{3}{|c|}{$40 \% \theta_{f}$} \\
\hline & $\begin{array}{c}\text { 上旬 } \\
1-10 \mathrm{~d}\end{array}$ & $\begin{array}{c}\text { 中旬 } \\
11-20 \mathrm{~d}\end{array}$ & $\begin{array}{c}\text { 下旬 } \\
21-31 \mathrm{~d}\end{array}$ & $\begin{array}{c}\text { 上旬 } \\
1-10 \mathrm{~d}\end{array}$ & $\begin{array}{c}\text { 中旬 } \\
11-20 \mathrm{~d}\end{array}$ & $\begin{array}{c}\text { 下旬 } \\
21-31 \mathrm{~d}\end{array}$ & $\begin{array}{c}\text { 上旬 } \\
1-10 \mathrm{~d}\end{array}$ & $\begin{array}{c}\text { 中旬 } \\
11-20 \mathrm{~d}\end{array}$ & $\begin{array}{c}\text { 下旬 } \\
21-31 \mathrm{~d}\end{array}$ \\
\hline 3 月 March & 0.01 & 0.033 & 0.15 & 0.01 & 0.045 & 0.05 & 0.006 & 0.019 & 0.015 \\
\hline 4 月 April & 0.342 & 0.412 & 0.466 & 0.238 & 0.5 & 0.371 & 0.117 & 0.352 & 0.352 \\
\hline 5月 May & 1.759 & 2.282 & 2.275 & 1.359 & 1.946 & 1.432 & 1.215 & 1.211 & 1.30 \\
\hline 6月 June & 2.296 & 2.299 & 2.477 & 1.802 & 1.884 & 1.968 & 1.254 & 1.358 & 1.397 \\
\hline 7月 July & 2.54 & 2.66 & 2.482 & 2.018 & 1.748 & 1.599 & 1.137 & 1.352 & 1.241 \\
\hline 8 月 August & 2.213 & 1.894 & 2.005 & 1.213 & 1.136 & 1.237 & 0.957 & 0.866 & 0.828 \\
\hline 9 月 September & 1.808 & 0.971 & 0.705 & 1.172 & 0.72 & 0.505 & 0.831 & 0.59 & 0.367 \\
\hline 10 月 October & 1.313 & 0.38 & - & 0.961 & 0.26 & - & 0.717 & 0.167 & - \\
\hline
\end{tabular}

表 33 种土壤水分下杨树总耗水量、总生物量及总 WUE 的比较

Table 3 Total water consumption, biomass, water use efficiency of Populus simonii under different soil water stress

\begin{tabular}{cccc}
\hline $\begin{array}{c}\text { 土壤含水率 } \\
\text { Soil water content }\end{array}$ & $\begin{array}{c}\text { 耗水量 }(\mathrm{kg}) \\
\text { Water consumption }\end{array}$ & $\begin{array}{c}\text { 生物量 }(\mathrm{g}) \\
\text { Biomass }\end{array}$ & $\begin{array}{c}\text { 水分利用率 }\left(\mathrm{g} \mathrm{kg}{ }^{-1}\right) \\
\text { Water use efficiency }\end{array}$ \\
\hline $70 \% \theta_{f}$ & $26.34^{\mathrm{a} \mathrm{A}}$ & $155.0^{\mathrm{a} \mathrm{A}}$ & $5.88^{\mathrm{b} B}$ \\
$55 \% \theta_{f}$ & $16.46^{\mathrm{b} \mathrm{B}}$ & $100.2^{\mathrm{b} \text { B }}$ & $6.11^{\mathrm{aA}}$ \\
$40 \% \theta_{f}$ & $9.88^{\mathrm{c} \mathrm{C}}$ & $46.78^{\mathrm{c} \mathrm{C}}$ & $4.76^{\mathrm{C}}$ \\
\hline 相同字母不显著 小写为 $\alpha=0.05$ 水平 & 大写为 $\alpha=0.01$ 水平 $(S S R$ 检验 $)$ & The \\
\hline
\end{tabular}

相同子母不显著 小写为 $\alpha=0.05$ 皮
and capital letter means $\alpha=0.01$ ( SSR test)

树 8 月 (图 7d) 的耗水日变化在适宜水分和中度干 旱下均为双峰曲线, 其两个高峰分别在 10:00 左右 和 $15: 00$ 左右, 而在严重干旱下的耗水高峰是在 10:00 14:00 之间, 为单峰曲线; 夜间耗水达最低 值。9月 (图 7e) 3 种处理的耗水日进程为单峰曲 线, 其耗水高峰在 12:00 左右。

从上述结果可以看出，杨树在同一种土壤水分 条件下不同的测定日, 其耗水高峰出现的时间不同， 而在同一测定日中, 不同土壤水分下杨树耗水日进 程不同, 随着土壤水分含量的不同 杨树日耗水高峰 出现的时间发生相应的变化。

2.5 不同土壤水分含量对杨树单株总耗水量、总生 物量及总 WUE 的影响

从表 3 可以看出 杨树整个生长季的总耗水量、 总生物量的大小均为适宜水分 > 中度干旱 > 严重干 旱。其差异性均达极显著水平。在适宜水分下，总 生物量是中度干旱下的 1.55 倍, 是严重干旱下的 3.3 倍。而适宜水分下的总耗水量是中度干旱下的 1.6 倍, 是严重亏缺下的 2.7 倍, 但杨树的总 WUE 则 为中度干旱 > 适宜水分 > 严重干旱, 即中度干旱时 杨树的水分利用率为最高。

\section{3 讨 论}

叶水势是反映植物缺水最敏感的指标之一, 叶 含水量则可以直接反映植物体内的水分状况。韩孶
莲等(1991)研究证明随土壤含水量的下降, 沙棘叶 水势和叶含水量下降明显, 并证明沙棘具有低水势 耐旱特征。顾振瑜等 (1999) 证明元宝枫 (Acer truncatum) 具有低水势的耐旱特征。本研究证明干旱对 杨树叶水势和含水率有较大的影响，在中度水分亏 缺和严重干旱下杨树叶含水量和水势均有下降, 但 在严重干旱下杨树叶水势和叶含水量下降最为显 著和耐旱性较强的沙棘 (Hippophae rhamnoides)、油 松(Pinus tabalaeformis)、元宝枫等植物相比, 目前在 黄土高原广泛栽植的杨树并不具有低水势的耐旱特 征。

在适宜水分和中度亏缺的土壤水分条件下，杨 树的快速生长时间和干物质积累均在 5 6 月 , 在严 重干旱下杨树生长没有表现出生长大周期的特征, 曲线比较平缓, 表现出累积性生长曲线, 其干物质积 累主要在 5 月, 杨树在 3 种土壤水分条件下生长速 率和干物质积累的大小顺序是适宜水分 $>$ 中度干旱 > 严重干旱。在黄土高原, 有相当多的人工林土壤 含水量处于田间持水量的 $30 \%$ 以下, 而大部分的降 雨又主要集中在 7 9 月, 在 5 6 月的降水不能满 足杨树快速生长所需要的水分条件, 导致杨树生长 不良，出现了大片的低产林，因此，在黄土高原的大 部分地区水分较差的立地条件不能栽植杨树，只能 在局部水分较好的立地条件下栽植。

有研究证明土壤干旱会导致植物光合速率、蒸 

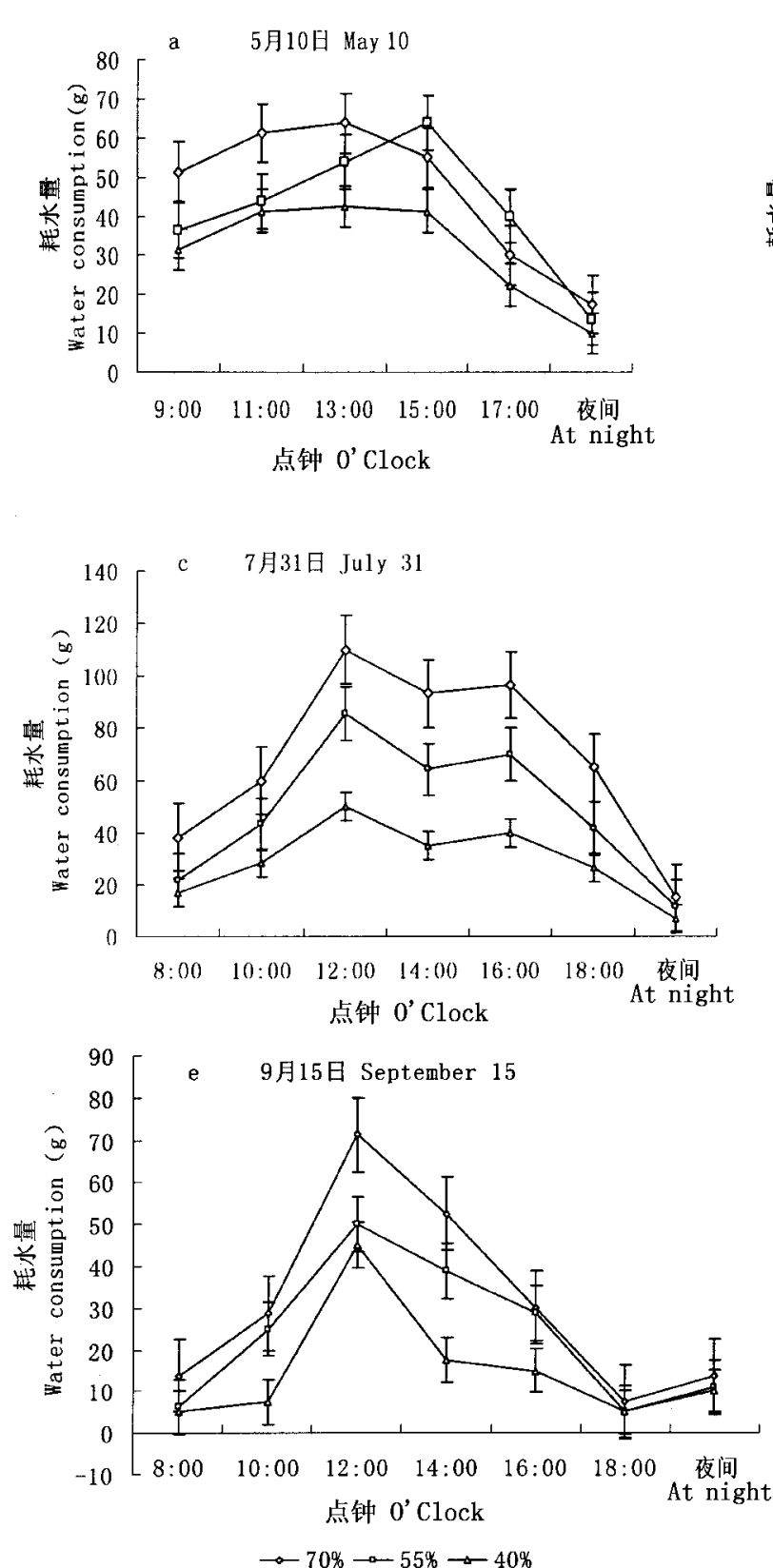
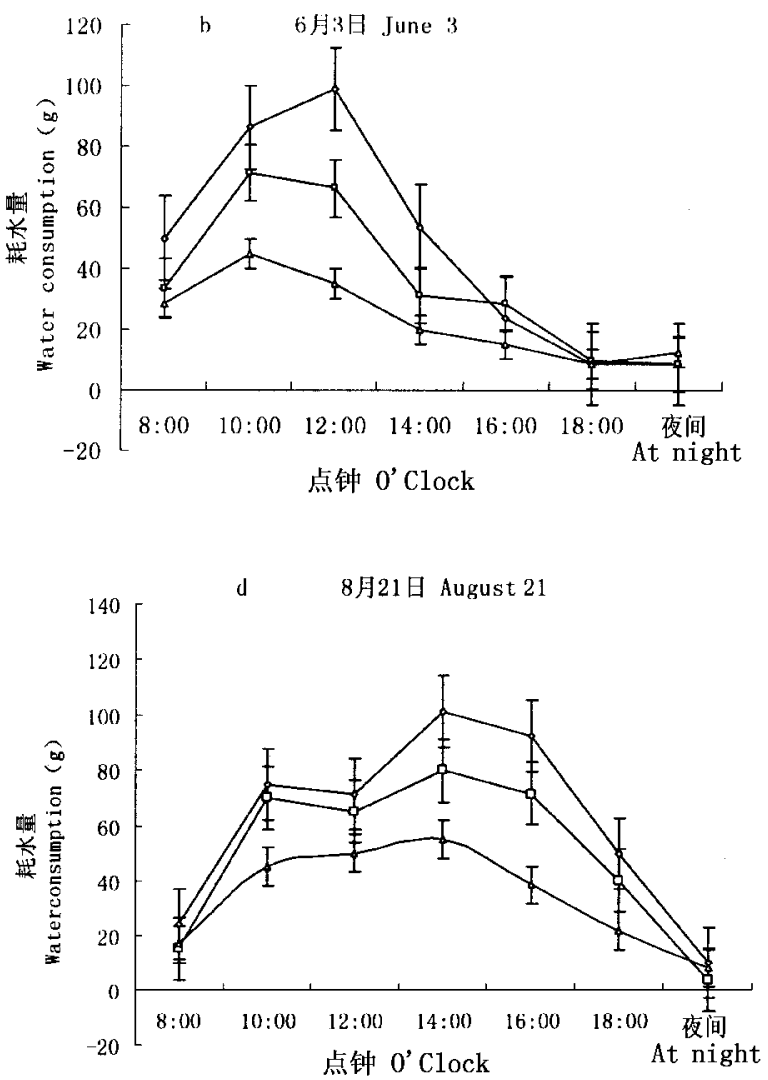

图 73 种土壤水分下杨树在各月的耗水日进程

Fig.7 Changes of water consumption of Populus simonii on May 10 June 3, July 31 ,August 21 and September 15 under three soil water stress

腾速率均下降,由于蒸腾速率下降幅度大光合速率 下降幅度小,使单叶水平上的 WUE 升高 (Thomas， 1986 ; 接玉玲等 ,2001)，从我们对杨树各月的测定结 果来看,杨树在不同土壤干旱下,单叶水平上的 $W U E$ 和 $T_{r}$ 并不符合此规律。随着土壤干旱的加 剧 杨树的光合速率和单叶 WUE 也随之下降, 其顺 序是适宜水分 > 中度干旱 > 严重干旱。在 3 种土 壤水分条件下,杨树的总 WUE 则表现为中度干旱 高于其它两种水分处理, 这表明在土壤水分从适宜 水分到中度干旱的变化过程中, 杨树消耗单位水量
所累积干物质的量增加, 而在从中度干旱到严重干 旱的变化过程中，杨树消耗单位水量所累积干物质 的量减少。在我国西北半干旱地区、黄土高原森林 草原过渡区的大部分立地条件下, 土壤水分经常处 于中度干旱到严重干旱的变化过程中, 不能满足杨 树累积更多干物质所需水量。

依据上述研究结果，对于杨树在西北干旱、半干 旱地区造林时, 要特别注意当地土壤水分是否能达 到杨树的生长对水分的要求, 尤其是黄土高原的降 雨主要集中在 $7 \sim 9$ 月，而杨树最高月耗水量主要是 
在 $6 \sim 7$ 月, 新枝条快速生长时期在 $5 \sim 6$ 月, 因此, 在该地区雨季到来之前, 黄土高原的土壤水分不能 满足杨树生长对水分的需要。所以, 对于杨树这种 耗水量大、抗旱性差的速生树种不适宜大面积栽植 在黄土高原地区, 适合栽植在阴坡、沟道等水分条件 较好的立地条件下。以上是对杨树在不同土壤干旱 下的生长、水分利用、干物质累积、耗水量等生理生 态过程的认识及结论, 它将为在三北地区人工林建 设过程中的树种选择与合理配置提供参考。

\section{参 考 文 献}

Bo, J. B. (卜基保). 1997. Effect of site factors on poplar growth in plains of the Changjiang River. Journal of Zhejiang Forestry College(浙江林学院学报), 14:298 302. (in Chinese with English abstract)

Chu, Z. W. (褚作旺), Y. Sun (孙勇), H. Y. Guo (郭洪 印), Q. Guo (郭全) \& H. W. Yuan (袁洪伟). 2003. Investigation of lower-yield stand poplar. Protection Forest Science and Technology (防护林科技), 3: $31 \sim 32$. (in Chinese with English abstract)

Gao, J. (高健) \& D. G. Huang (黄大国). 2002. Study of ecophysiological factors affecting poplar tree photosynthesis on the Yangtze River beach land. Journal of Central South Forestry University (中南林学院学报), 22 (2): 40 43. (in Chinese with English abstract)

$\mathrm{Gu}, \mathrm{Z} . \mathrm{Y}$. (顾振瑜), J. J. Hu (胡景江), J. L. Wen (文建 雷) \& S. Q. Wang (王姝清). 1999. A study on adaptability of maple to drought stress. Journal of Northwest Forestry University (西北林学院学报), 14(2) :1 6. (in Chinese with English abstract)

Han, R. L. (韩芯莲), Z. S. Liang (梁宗锁) \& H. Y. Zou (邹厚远). 1991. Water consumption properties of Chinese seabuckthron under different soil drought. Hippophae (沙棘), 4:33 35, 38. (in Chinese with English abstract)

Hou, Q. C. (侯庆春) \& R. L. Han (韩芯莲). 2000. Problems on vegetation construction in loess plateau region. Bulletin of Soil and Water Conservation(水土保持通报), 20(2) : 53 56. (in
Chinese with English abstract)

Hu, X. S. (胡新生), J. W . Liu (刘建伟) \& S. J. Wang (王 世绩). 1997. Comparison of the net photosynthesis rate of four poplar clones at different temperature and humidity regimes. Scientia Silvae Sinicae(林业科学), 33(2) : $107 \sim 115$. (in Chinese with English abstract)

Jia，L. M. (贾黎明)，Y. K. Wei (韦艳葵)，Y. A. Li (李延 安), L. Yang (杨丽) \& C. S. Xing (邢长山). 2004. The growth and photosynthesis of poplar trees in fast-growing and high-yield plantations with subterranean drip irrigation. Scientia Silvae Sinicae (林业科学), 40(2): 61 67. (in Chinese with English abstract)

Jie, Y. L. (接玉玲), H. Q. Yang (杨洪强), M. G. Cui (崔 明刚) \& X. S. Luo (罗新书). 2001. Relationship between soil water content and water use efficiency of apple leaves. Chinese Journal of Applied Ecology (应用生态学报)，12: 387 390. (in Chinese with English abstract)

Ju, G. S. (巨关升), S. K. Zheng (郑世锴)，W. Z. Zhao (赵 文忠), W. K. Hu (胡万库) \& Y. J. Liu (刘永军). 2000. Study on the high-yield poplar cultivating pattern in middle-temperate zone plantation. Scientia Silvae Sinicae (林业科学), 36 (4) : $92 \sim 96$. (in Chinese with English abstract)

Li, H. J. (李洪建) , B. F. Chai (柴宝峰) \& M. B. Wang(王 孟本). 2000. Study on the water physio-ecological characteristics of Populus beijingensis. Acta Ecologica Sinica (生态学报), 20:417 422. (in Chinese with English abstract)

Liu, F. J. ( 刘奉觉) \& S. K. Zheng (郑世锴). 1991. Study on water physiology of poplar. Beijing: Beijing Agriculture University Press.

Liu, Y. R. (刘雅荣), F. J. Liu (刘奉觉) \& S. Wang (王爽). 1983. A study on the growth and photosynthesis of 4 poplars. Scientia Silvae Sinicae (林业科学), 19:269 276. (in Chinese with English abstract)

Thomas, H. 1986. Characteristics of Dactylis glomerata L., Lolium perenne L. and L. multiflorum Lam. plants. Annals of Botany, 57: $211 \sim 223$.

Zeng, F. J. (曾凡江) \& X. Song (宋轩). 2000. A review on the eco-physiological study of poplars in oasis and its prospect. Chinese Journal of Applied Ecology (应用生态学报), 11: 780 784. (in Chinese with English abstract) 